\title{
Generation of entangled states for many multi-level atoms in a thermal cavity and ions in thermal motion
}

\author{
Shi-Biao Zheng \\ Department of Electronic Science and Applied Physics \\ Fuzhou University \\ Fuzhou 350002, P. R. China
}

(Dated: August 4, 2018)

\begin{abstract}
We propose a scheme for generating entangled states for two or more multi-level atoms in a thermal cavity. The photon-number dependent parts in the effective Hamiltonian are canceled with the assistant of a strong classical field. Thus the scheme is insensitive to both the cavity decay and the thermal field. The scheme does not require individual addressing of the atoms in the cavity. The scheme can also be used to generate entangled states for many hot multi-level ions.
\end{abstract}

PACS numbers: PACS number: 42.50.Dv, 42.50.Vk, 03.65.Bz

Entanglement of two or more particles is not only of significance for test of quantum mechanics against local hidden theory [1-3], but also useful in quantum cryptography [4] and quantum teleportation [5]. Most of research in quantum nonlocality and quantum information is based on entanglement of two-level particles. Entangled states for two-level particles have been observed for photons [6-8], atoms in cavity QED [9-11], and ions in a trap [12-14].

Recently, it has been shown that violations of local realism by two entangled $\mathrm{N}$-dimensional systems are stronger than for two qubits [15]. The GreenbergerHorne-Zeiliner paradox has also been extended to the many N-dimensional systems [16]. Furthermore, it has been shown that quantum cryptography based on entangled qutrits is more secure than that based on entangled qubits [17]. High-dimensional entanglement for photons has been observed [18-21]. However, there have no reports on the realization of entanglement for multi-level massive particles. Recently, Zou et al. [22] have proposed a scheme for the generation of entangled states for two three-level atoms in cavity QED using nonresonant interaction of two atoms with a cavity [23]. The scheme is insensitive to cavity decay. The main drawback of the scheme is that it requires individual addressing of the atoms when both atoms are still in the cavity, which is experimentally problematic.

In this paper we propose a scheme for generating entangled states for many multi-level atoms in cavity QED and ions in a trap. In cavity QED, our scheme does not require individual addressing of the atoms in the cavity. Another distinct feature of the present scheme is that the photon-number dependent parts in the effective Hamiltonian are canceled with the assistant of a strong classical driving field. Due to this feature the scheme is insensitive to both the cavity decay and thermal field. For the trapped ions, our scheme is insensitive to the thermal motion.

We consider $\mathrm{N}$ identical ladder-type three-level atoms simultaneously interacting with a single-mode cavity field and driven by a classical field. The atomic states are denoted by $|g\rangle,|e\rangle$, and $|i\rangle$. The transition frequency between the states $|e\rangle$ and $|i\rangle$ is highly detuned from the cavity frequency and thus the state $|i\rangle$ is not affected during the atom-cavity interaction. The Hamiltonian is (assuming $\hbar=1$ ) [24,25]

$$
\begin{gathered}
H=\omega_{0} \sum_{j=1}^{N} S_{z, j}+\omega_{a} a^{+} a+\sum_{j=1}^{N}\left[g\left(a^{+} S_{j}^{-}+a S_{j}^{+}\right)\right. \\
\left.+\Omega\left(S_{j}^{+} e^{-i \omega t}+S_{j}^{-} e^{i \omega t}\right)\right]
\end{gathered}
$$

where $\mathrm{S}_{j}^{+}=\left|e_{j}\right\rangle\left\langle g_{j}\left|, \mathrm{~S}_{j}^{-}=\right| g_{j}\right\rangle\left\langle e_{j}\right|, S_{z, j}=\frac{1}{2}\left(\left|e_{j}\right\rangle\left\langle e_{j}\right|-\right.$ $\left.\left|g_{j}\right\rangle\left\langle g_{j}\right|\right)$, with $\left|e_{j}\right\rangle$ and $\left|g_{j}\right\rangle(\mathrm{j}=1,2)$ being the excited and ground states of the jth atom, $\mathrm{a}^{+}$and a are the creation and annihilation operators for the cavity mode, and $g$ is the atom-cavity coupling strength, and $\Omega$ is the Rabi frequency of the classical field. Assume that $\omega_{0}=$ $\omega$. Then the interaction Hamiltonian, in the interaction picture, is

$$
H_{i}=\sum_{j=1}^{N}\left[g\left(e^{-i \delta t} a^{+} S_{j}^{-}+e^{i \delta t} a S_{j}^{+}\right)+\Omega\left(S_{j}^{+}+S_{j}^{-}\right)\right],
$$

$\delta$ is the detuning between the atomic transition frequency $\omega_{0}$ and cavity frequency $\omega$. Define the new atomic basis

$$
\left|+{ }_{j}\right\rangle=\frac{1}{\sqrt{2}}\left(\left|g_{j}\right\rangle+\left|e_{j}\right\rangle\right), \quad\left|{ }_{j}\right\rangle=\frac{1}{\sqrt{2}}\left(\left|g_{j}\right\rangle-\left|e_{j}\right\rangle\right) .
$$

Then we can rewrite $H_{i}$ as

$$
\begin{array}{r}
H_{i}=\sum_{j=1}^{N}\left[g e^{-i \delta t} a^{+}\left(\sigma_{z, j}+\frac{1}{2} \sigma_{j}^{+}-\frac{1}{2} \sigma_{j}^{-}\right)\right. \\
\left.+e^{i \delta t} a\left(\sigma_{z, j}+\frac{1}{2} \sigma_{j}^{-}-\frac{1}{2} \sigma_{j}^{+}\right)+2 \Omega \sigma_{z, j}\right],
\end{array}
$$

where $\sigma_{z, j}=\frac{1}{2}\left(\left|+{ }_{j}\right\rangle\left\langle+{ }_{j}|-|{ }_{j}\right\rangle\left\langle{ }_{j}\right|\right), \sigma_{j}^{+}=\left|+{ }_{j}\right\rangle\left\langle{ }_{j}\right|$ and $\sigma_{j}^{-}=\left|-{ }_{j}\right\rangle\left\langle+{ }_{j}\right|$.

The time evolution of this system is decided by Schrödinger's equation:

$$
i \frac{d|\psi(t)\rangle}{d t}=H_{i}|\psi(t)\rangle
$$


Perform the unitary transformation

$$
|\psi(t)\rangle=e^{-i H_{0} t}\left|\psi^{\prime}(t)\right\rangle,
$$

with

$$
H_{0}=2 \Omega \sum_{j=1}^{N} \sigma_{z, j}
$$

Then we obtain

$$
i \frac{d\left|\psi^{\prime}(t)\right\rangle}{d t}=H_{i}^{\prime}\left|\psi^{\prime}(t)\right\rangle
$$

where

$$
\begin{array}{r}
H_{i}^{\prime}=\sum_{j=1,2}\left[g e^{-i \delta t} a^{+}\left(\sigma_{z, j}+\frac{1}{2} \sigma_{j}^{+} e^{2 i \Omega t}-\frac{1}{2} \sigma_{j}^{-} e^{-2 i \Omega t}\right)\right. \\
+e^{i \delta t} a\left(\sigma_{z, j}+\frac{1}{2} \sigma_{j}^{-} e^{-2 i \Omega t}-\frac{1}{2} \sigma_{j}^{+} e^{2 i \Omega t}\right) .
\end{array}
$$

Assuming that $2 \Omega \gg \delta, g$, we can neglect the terms oscillating fast. Then $H_{i}^{\prime}$ reduces to

$$
\begin{aligned}
H_{i}^{\prime} & =\sum_{j=1}^{N} g\left(e^{-i \delta t} a^{+}+e^{i \delta t} a\right) \sigma_{z, j} \\
& =\frac{1}{2} \sum_{j=1}^{N} g\left(e^{-i \delta t} a^{+}+e^{i \delta t} a\right)\left(S_{j}^{+}+S_{j}^{-}\right) .
\end{aligned}
$$

In the case $\delta \gg g / 2$, there is no energy exchange between the atomic system and the cavity. The resonant transitions are $\left|e_{j} g_{k} n\right\rangle \longleftrightarrow\left|g_{j} e_{k} n\right\rangle$ and $\left|e_{j} e_{k} n\right\rangle \longleftrightarrow$ $\left|g_{j} g_{k} n\right\rangle$. The transition $\left|e_{j} g_{k} n\right\rangle \longleftrightarrow\left|g_{j} e_{k} n\right\rangle$ is mediated by $\left|g_{j} g_{k} n \pm 1\right\rangle$ and $\left|e_{j} e_{k} n \pm 1\right\rangle$. The contributions of $\left|g_{j} g_{k} n \pm 1\right\rangle$ are equal to those of $\left|e_{j} e_{k} n \pm 1\right\rangle$. The corresponding Rabi frequency is given by

$$
2 \frac{\left\langle e_{j} g_{k} n\left|H_{i}^{\prime}\right| g_{j} g_{k} n+1\right\rangle\left\langle g_{j} g_{k} n+1\left|H_{i}^{\prime}\right| g_{j} e_{k} n\right\rangle}{\delta}
$$

$$
+2 \frac{\left\langle e_{j} g_{k} n\left|H_{i}^{\prime}\right| g_{j} g_{k} n-1\right\rangle\left\langle g_{j} g_{k} n-1\left|H_{i}^{\prime}\right| g_{j} e_{k} n\right\rangle}{-\delta}=\frac{g^{2}}{2 \delta} \text {. }
$$

Since the transition paths interfere destructively the Rabi frequency is independent of the photon-number of the cavity mode. The destructive interference of transition amplitudes was first proposed for trapped ions [26, 27]. The Rabi frequency for $\left|e_{j} e_{k} n\right\rangle \longleftrightarrow\left|g_{j} g_{k} n\right\rangle$, mediated by $\left|e_{j} g_{k} n \pm 1\right\rangle$ and $\left|g_{j} e_{k} n \pm 1\right\rangle$, is also equal to $g^{2} /(2 \delta)$. The Stark shift for the state $\left|e_{j}\right\rangle$ is

$$
\begin{array}{r}
\frac{\left\langle e_{j} n\left|H_{i}^{\prime}\right| g_{j} n+1\right\rangle\left\langle g_{j} n+1\left|H_{i}^{\prime}\right| e_{j} n\right\rangle}{\delta} \\
+\frac{\left\langle e_{j} n\left|H_{i}^{\prime}\right| g_{j} n-1\right\rangle\left\langle g_{j} n-1\left|H_{i}^{\prime}\right| e_{j} n\right\rangle}{-\delta}=\frac{g^{2}}{4 \delta} .
\end{array}
$$

The Stark shift for $\left|g_{j}\right\rangle$ is also $g^{2} /(4 \delta)$. The strong classical field induces the terms $g\left(e^{-i \delta t} a^{+} S_{j}^{+}+e^{i \delta t} a S_{j}^{-}\right)$, which result in the photon-number dependent Stark shifts negative to those induced by $g\left(e^{-i \delta t} a^{+} S_{j}^{-}+e^{i \delta t} a S_{j}^{+}\right)$. Thus the photon-number dependent Stark shifts are also cancelled. Then the effective Hamiltonian is given by

$$
\begin{array}{r}
H_{e}=\lambda\left[\frac{1}{2} \sum_{j=1}^{N}\left(\left|e_{j}\right\rangle\left\langle e_{j}|+| g_{j}\right\rangle\left\langle g_{j}\right|\right)\right. \\
\left.+\sum_{j, k=1}^{N}\left(S_{j}^{+} S_{k}^{+}+S_{j}^{+} S_{k}^{-}+H . c .\right)\right], j \neq k
\end{array}
$$

where $\lambda=\frac{g^{2}}{2 \delta}$. The distinct feature of the effective Hamiltonian is that it is independent of the photon-number of the cavity field. Without the strong classical field, the Stark shift terms are proportional to the photon number, and the terms $S_{j}^{+} S_{k}^{+}+H . c$ do not exist. The evolution operator of the system is given by

$$
U(t)=e^{-i H_{0} t} e^{-i H_{e} t}
$$

We note the atomic state evolution operator $U(t)$ is independent of the cavity field state, allowing it to be in a thermal state.

We first consider the case that $\mathrm{N}=2$. Assume the two atoms are initially in the state $\left|g_{1}\right\rangle\left|g_{2}\right\rangle$. After an interaction time $t_{1}$ the state of the system is

$$
\begin{array}{r}
\left|g_{1}\right\rangle\left|g_{2}\right\rangle \longrightarrow e^{-i \lambda t_{1}}\left\{\cos \left(\lambda t_{1}\right)\left[\cos \Omega t_{1}\left|g_{1}\right\rangle-i \sin \Omega t_{1}\left|e_{1}\right\rangle\right]\right] \\
\left.\left[\cos \Omega t_{1}\left|g_{2}\right\rangle-i \sin \Omega t_{1}\left|e_{2}\right\rangle\right]\right] \\
\left.-i \sin \left(\lambda t_{1}\right)\left[\cos \Omega t_{1}\left|e_{1}\right\rangle-i \sin \Omega t_{1}\left|g_{1}\right\rangle\right]\right] \\
\left.\left[\cos \Omega t_{1}\left|e_{2}\right\rangle-i \sin \Omega t_{1}\left|g_{2}\right\rangle\right]\right\}
\end{array}
$$

Choose the interaction time $t_{1}$ and Rabi frequency $\Omega$ appropriately so that $\sin \left(\lambda t_{1}\right)=1 / \sqrt{3}$ and $\Omega t_{1}=k \pi$, with $\mathrm{k}$ being an integer. Then we have

$$
\left|g_{1}\right\rangle\left|g_{2}\right\rangle \longrightarrow e^{-i \lambda t_{1}}\left\{\sqrt{\frac{2}{3}}\left|g_{1}\right\rangle\left|g_{2}\right\rangle-i \frac{1}{\sqrt{3}}\left|e_{1}\right\rangle\left|e_{2}\right\rangle\right\} .
$$

Now we switch off the classical field tuned to the $|g\rangle \rightarrow|e\rangle$, and switch on another classical field tuned to the $|e\rangle \rightarrow|f\rangle$. Choosing the Rabi frequency and interaction time appropriately so that the atoms undergoes the transitions: $|e\rangle \rightarrow|f\rangle$. We here assume that this classical field is sufficiently strong and thus the interaction time is so short that the dispersive atom-cavity interaction can be neglected during the application of this classical field. This leads to

$$
e^{-i \lambda t_{1}}\left\{\sqrt{\frac{2}{3}}\left|g_{1}\right\rangle\left|g_{2}\right\rangle-i \frac{1}{\sqrt{3}}\left|f_{1}\right\rangle\left|f_{2}\right\rangle\right\} .
$$

Then we again switch on the classical field tuned to the $|g\rangle \rightarrow|e\rangle$, and switch off the field tuned to the $|e\rangle \rightarrow|f\rangle$. 
The Hamiltonian is again given by Eq.(13). After another interaction time $t_{2}$. We obtain

$$
\begin{array}{r}
e^{-i \lambda\left(t_{1}+t_{2}\right)} \sqrt{\frac{2}{3}}\left\{\cos \left(\lambda t_{2}\right)\left[\cos \Omega^{\prime} t_{2}\left|g_{1}\right\rangle-i \sin \Omega^{\prime} t_{2}\left|e_{1}\right\rangle\right]\right. \\
{\left[\cos \Omega^{\prime} t_{2}\left|g_{2}\right\rangle-i \sin \Omega^{\prime} t_{2}\left|e_{2}\right\rangle\right]} \\
-i \sin \left(\lambda t_{2}\right)\left[\cos \Omega^{\prime} t_{2}\left|e_{1}\right\rangle-i \sin \Omega^{\prime} t_{2}\left|g_{1}\right\rangle\right] \\
\left.\left[\cos \Omega^{\prime} t_{2}\left|e_{2}\right\rangle-i \sin \Omega^{\prime} t_{2}\left|g_{2}\right\rangle\right]\right\} \\
-i e^{-i \lambda t_{1}} \frac{1}{\sqrt{3}}\left|f_{1}\right\rangle\left|f_{2}\right\rangle,
\end{array}
$$

where $\Omega^{\prime}$ is the Rabi frequency of the classical field during the interaction time $t_{2}$. Choose the interaction time $t_{2}$ and Rabi frequency $\Omega^{\prime}$ appropriately so that $\lambda t_{2}=\pi / 4$ and $\Omega^{\prime} t_{2}=2 k^{\prime} \pi$,with $\mathrm{k}^{\prime}$ being an integer. Then we have

$e^{-i \lambda t_{1}} \sqrt{\frac{1}{3}}\left\{e^{-i \lambda t_{2}}\left|g_{1}\right\rangle\left|g_{2}\right\rangle-i e^{-i \lambda t_{2}}\left|e_{1}\right\rangle\left|e_{2}\right\rangle-i\left|f_{1}\right\rangle\left|f_{2}\right\rangle\right\}$.

This is a maximally entangled state for the two threelevel atoms. We here do not require individually addressing of the atoms when they are in the cavity. Furthermore, our scheme is not only insensitive to the cavity decay but also insensitive to the thermal photons. The thermal field gradually builds up during the operations [10]. Thus, our scheme is important in view of experiment.

We now turn to the problem of generating entanglement for three or more three-level atoms with a thermal cavity. The effective Hamiltonian $\mathrm{H}_{e}$ can also be rewritten as

$$
H_{e}=2 \lambda S_{x}^{2}
$$

where

$$
S_{x}=\frac{1}{2} \sum_{j=1}^{N}\left(S_{j}^{+}+S_{j}^{-}\right) .
$$

Assume that the atoms are initially in the state $\left|g_{1} g_{2} \ldots g_{N}\right\rangle$. Using the representation of the operator $\mathrm{S}_{z}$, the atomic state $\left|g_{1} g_{2} \ldots g_{N}\right\rangle$ and $\left|e_{1} e_{2} \ldots e_{N}\right\rangle$ can be expressed as $|N / 2,-N / 2\rangle$ and $|N / 2, N / 2\rangle$. On the other hand, such states can be expanded in terms of the eigenstates of $\mathrm{S}_{x}[25,26,28]$

$$
\begin{gathered}
|N / 2,-N / 2\rangle=\sum_{M=-N / 2}^{N / 2} C_{M}|N / 2, M\rangle_{x} . \\
|N / 2, N / 2\rangle=\sum_{M=-N / 2}^{N / 2} C_{M}(-1)^{N / 2-M}|N / 2, M\rangle_{x} .
\end{gathered}
$$

Thus, the evolution of the system is

$$
\sum_{M=-N / 2}^{N / 2} C_{M} e^{-2 i\left(\Omega M+\lambda M^{2}\right) t}|N / 2, M\rangle_{x} .
$$

When $\mathrm{N}$ is even $\mathrm{M}$ is an integer. With the choice $\lambda t=$ $\pi / 4$ and $\Omega t=n \pi$ we obtain

$$
\begin{aligned}
& \frac{1}{\sqrt{2}} \sum_{M=-N / 2}^{N / 2} C_{M}\left[e^{-i \pi / 4}+e^{i \pi / 4}(-1)^{M}\right]|N / 2, M\rangle_{x} \\
& =\frac{1}{\sqrt{2}}\left(e^{-i \pi / 4}\left|g_{1} g_{2} \ldots g_{N}\right\rangle+e^{i \pi / 4}(-1)^{N / 2}\left|e_{1} e_{2} \ldots e_{N}\right\rangle\right) .
\end{aligned}
$$

On the other hand, for the case that $\mathrm{N}$ is odd we choose $\lambda t=\pi / 4$ and $\Omega t=\left(2 n+\frac{3}{4}\right) \pi$. Then we obtain

$$
\frac{1}{\sqrt{2}} e^{i \frac{7}{8} \pi}\left[e^{-i \pi / 4}\left|g_{1} g_{2} \ldots g_{N}\right\rangle+e^{i \pi / 4}(-1)^{(1+N) / 2}\left|g_{1} g_{2} \ldots g_{N}\right\rangle\right] .
$$

By this way we obtain a multiatom Greenberger-HorneZeilinger state [2].

We here assume that $\mathrm{N}$ is even. After the state of Eq. (26) is prepared we switch off the classical field tuned to $|e\rangle \rightarrow|g\rangle$ and perform the transformation: $|e\rangle \rightarrow|f\rangle$. Then we have

$$
\frac{1}{\sqrt{2}}\left(e^{-i \pi / 4}\left|g_{1} g_{2} \ldots g_{N}\right\rangle+e^{i \pi / 4}(-1)^{N / 2}\left|f_{1} f_{2} \ldots f_{N}\right\rangle\right) .
$$

Then we again switch on the classical field tuned to $|e\rangle \rightarrow|g\rangle$. After another interaction time t we obtain an entangled state for the $\mathrm{N}$ three-level atoms

$$
\begin{gathered}
\frac{1}{2} e^{-i \pi / 4}\left(e^{-i \pi / 4}\left|g_{1} g_{2} \ldots g_{N}\right\rangle+e^{i \pi / 4}(-1)^{N / 2}\left|e_{1} e_{2} \ldots e_{N}\right\rangle\right) \\
+\frac{1}{\sqrt{2}} e^{i \pi / 4}(-1)^{N / 2}\left|f_{1} f_{2} \ldots f_{N}\right\rangle .
\end{gathered}
$$

After the $\mathrm{N}$ atoms exits the cavity we can prepare $\mathrm{N}-1$ atoms into a maximally entangled state via manipulating the Nth atom. We first perform the transformations:

$$
\begin{aligned}
& \left|g_{N}\right\rangle \rightarrow \frac{1}{\sqrt{2}}\left|g_{N}\right\rangle+\frac{1}{\sqrt{10}}\left|e_{N}\right\rangle-\sqrt{\frac{2}{5}}\left|f_{N}\right\rangle, \\
& \left|e_{N}\right\rangle \rightarrow-\frac{1}{\sqrt{2}}\left|g_{N}\right\rangle+\frac{1}{\sqrt{10}}\left|e_{N}\right\rangle-\sqrt{\frac{2}{5}}\left|f_{N}\right\rangle, \\
& \left|f_{N}\right\rangle \rightarrow \frac{2}{\sqrt{5}}\left|e_{N}\right\rangle+\sqrt{\frac{1}{5}}\left|f_{N}\right\rangle .
\end{aligned}
$$

Then we detect the state of the Nth atom. The detection of the state $\left|f_{N}\right\rangle$ collapses the N-1 atoms onto the maximally entangled state

$$
\begin{gathered}
\frac{1}{\sqrt{3}}\left(e^{-i \pi / 2}\left|g_{1} g_{2} \ldots g_{N-1}\right\rangle+(-1)^{N / 2}\left|e_{1} e_{2} \ldots e_{N-1}\right\rangle\right. \\
-e^{i \pi / 4}(-1)^{N / 2}\left|f_{1} f_{2} \ldots f_{N-1}\right\rangle
\end{gathered}
$$

The probability of success is 0.3 .

We note we can generate a maximally entangled state for $\mathrm{N}$ four-level atoms determinately. The fourth level 
is $|h\rangle$. After the atoms are prepared in the state of Eq. (28) we perform the transformation: $|g\rangle \longleftrightarrow|f\rangle$ and $|e\rangle \longleftrightarrow|h\rangle$. This leads to

$$
\begin{gathered}
\frac{1}{\sqrt{2}} e^{i \pi / 4}(-1)^{N / 2}\left|g_{1} g_{2} \ldots g_{N}\right\rangle+\frac{1}{2}\left(e^{-i \pi / 2}\left|f_{1} f_{2} \ldots f_{N}\right\rangle\right. \\
\left.+(-1)^{N / 2}\left|h_{1} h_{2} \ldots h_{N}\right\rangle\right) .
\end{gathered}
$$

Then we again switch on the classical field tuned to $|e\rangle \rightarrow|g\rangle$. After another interaction time t we obtain an entangled state for the $\mathrm{N}$ four-level atoms

$$
\begin{gathered}
\quad \frac{1}{2}\left[(-1)^{N / 2}\left|g_{1} g_{2} \ldots g_{N}\right\rangle+e^{i \pi / 2}\left|e_{1} e_{2} \ldots e_{N}\right\rangle\right. \\
\left.+e^{-i \pi / 2}\left|f_{1} f_{2} \ldots f_{N}\right\rangle+(-1)^{N / 2}\left|h_{1} h_{2} \ldots h_{N}\right\rangle\right] .
\end{gathered}
$$

We note the idea can also be used to the ion trap system. We consider that $\mathrm{N}$ ions are confined in a linear trap. Then we simultaneously excite the ions with two lasers of frequencies $\omega_{0}+\nu+\delta$ and $\omega_{0}-\nu-\delta$, where $\omega_{0}$ is the frequency of the transition $|e\rangle \rightarrow|g\rangle$ and $\nu$ is the frequency of the one collective vibrational mode. Suppose $\delta$ is much smaller than $\nu$ and thus we can neglect other vibrational modes. In this case the Hamiltonian for the system is given by $[26,27]$

$$
\begin{gathered}
\hat{H}=\nu \hat{a}^{+} \hat{a}+\omega_{0} \sum_{j=1}^{N} \hat{S}_{z, j}+\left\{\Omega e^{-i \phi} \sum_{j=1}^{N} \hat{S}_{j}^{+} e^{i \eta\left(\hat{a}^{+}+\hat{a}\right)}\right. \\
\left.\left[e^{-i\left(\omega_{0}+\nu+\delta\right) t}+e^{-i\left(\omega_{0}-\nu-\delta\right) t}\right]+H . c .\right\}
\end{gathered}
$$

where $\stackrel{\wedge}{a}^{+}$and $\hat{a}$ are the creation and annihilation operators for the collective vibrational mode, and $\eta=$ $k / \sqrt{2 \nu M}$ is the Lamb-Dicke parameter with $\mathrm{k}$ being the wavevector along the trap axis and $M$ the mass of the ion collection. We here have assumed the lasers have the same Rabi frequency $\Omega$, phase $\phi$ and same wavevector $\mathrm{k}$. Furthermore, we consider the resolved sideband regime, where the vibrational frequency $\nu$ is much larger than other characteristic frequencies of the problem. In this case we discard the rapidly oscillating terms and obtain the Hamiltonian in the interaction picture

$$
\begin{gathered}
\stackrel{\wedge}{H}=\Omega e^{-\eta^{2} / 2} e^{-i \phi} \sum_{j=1}^{N} \hat{S}_{j}^{+} \sum_{j=0}^{\infty} \frac{(i \eta)^{2 j+1}}{j !(j+1) !} \\
{\left[\hat{a}^{+(j+1)} \wedge^{j} e^{-i \delta t}+\hat{a}^{+j}{ }_{a}^{j+1} e^{i \delta t}\right]+H . c .}
\end{gathered}
$$

In the Lamb-Dicke regime, i.e., $\eta \sqrt{n+1} \ll 1$ with $\mathrm{n}$ being the phonon number, the Hamiltonian of Eq.(34) can be approximated by the expansion to the first order in $\eta$

$$
\stackrel{\wedge}{H}=i \eta \Omega e^{-i \phi} \sum_{j=1}^{N} \hat{S}_{j}^{+}\left(\hat{a}^{+} e^{-i \delta t}+\hat{a} e^{i \delta t}\right)+H . c .
$$

When $\delta \gg \eta \Omega$ and $\phi=\pi / 2$ the effective Hamiltonian has the same form as Eq. (13), with $\lambda=2 \Omega^{2} \eta^{2} / \delta$. For the case $\mathrm{N}=2$ we focus the two lasers on the ions for a time $\arcsin (1 / \sqrt{3}) / \lambda$, then perform the transformation: $|e\rangle \rightarrow|f\rangle$ with $|f\rangle$ being another internal state, followed by the application of the above mentioned two lasers for a time $\pi /(4 \lambda)$. The two ions are prepared in the state of Eq. (19). Using the procedure similar to that for cavity QED we can also generate entangled states for many multi-level ions. The effective Hamiltonian does not involve the external degree of freedom and thus the scheme is insensitive to the external state, allowing the it to be in a thermal state. For the generation of the states of Eq. (19), (28), and (32), we do not require individual addressing of the ions.

In conclusion, we have proposed a scheme for generating entangled states for two or more multi-level particles in both cavity QED and ion trap. In cavity QED, our scheme does not require individual addressing of atoms in the cavity. In cavity QED the scheme is insensitive to both cavity decay and thermal field, which is of importance from the experimental point of view. In ion trap, our scheme is insensitive to the thermal motion. Based on the experiments reported in Refs. [10], [13], and [14], our scheme is realizable with techniques presently available.

This work was supported by Fok Ying Tung Education Foundation 81008, the National Fundamental Research Program Under Grant No. 2001CB309300, the National Natural Science Foundation of China under Grant Nos. 60008003 and 10225421.
[1] J. S. Bell, Physic 1 (1965) 195.

[2] D. M. Greenberger, M. A. Horne, and A. Zeilinger, in Bell,s Theorem, Quantum Theory, and Conceptions of the Universe, edited by M.Kafatos (Kluwer, Dordrecht, 1989); D. M. Greenberger, M. A. Horne, A. Shimony, and A. Zeilinger, Am. J. Phys. 58, 1131 (1990).

[3] N. D. Mermin, Phys. Rev. Lett. 65, 1838 (1990).

[4] A. K. Ekert, Phys. Rev. Lett. 67, 661 (1991).

[5] C. H. Bennett et al., Phys. Rev. Lett. 70, 1895 (1993).
[6] D. Bouwmeester et al., Phys. Rev. Lett. 82, 1345 (1999).

[7] J-W. Pan et al., Nature 403, 515 (2000).

[8] J-W. Pan et al., Phys. Rev. Lett. 86, 4435 (2001).

[9] E. Hagley et al., Phys. Rev. Lett. 79, 1 (1997).

[10] S. Osnaghi et al., Phys. Rev. Lett. 87, 037902 (2001).

[11] A. Rauschenbeutel et al., Science 288, 2024 (2000).

[12] Q. A. Turchette et al., Phys. Rev. Lett. 81, 3631 (1998).

[13] M. A. Rowe et al., Nature 409, 791 (2001).

[14] C. A. Sackett et al., Nature 404, 256 (2000). 
[15] D. Kaszlikowski et al., Phys. Rev. Lett. 85, 4418 (2000).

[16] N. J. Cerf et al., Phys. Rev. Lett. 89, 080402 (2002).

[17] T. Durt et al., quant-ph/0207057

[18] A. Mair et al., Nature 412, 313 (2001).

[19] A. Lamas-Linares et al., Nature 412, 887 (2001).

[20] Hugues de Riedmatten et al., quant-ph/0204165

[21] J. C. Howell et al., Phys. Rev. Lett. 88, 030401 (2002).

[22] X. Zou et al., Phys. Rev. A 67, 044301 (2003).

[23] S. B. Zheng and G. C. Guo, Phys. Rev. Lett. 85, 2392 (2000).
[24] E. Solano et al., Phys. Rev. Lett. 90, 027903 (2003).

[25] S. B. Zheng, Phys. Rev. A 66, 060303 (R) (2002).

[26] A. S $\phi$ rensen and K. M $\phi$ lmer, Phys. Rev. Lett. 82, 1971 (1999); K. M $\phi$ lmer and A. S $\phi$ rensen, Phys. Rev. Lett. 82 (1999) 1835.

[27] E. Solano, R. L. de Matos, and N. Zagury, Phys. Rev. A 59, R2539 (1999).

[28] D. M. Brink and G. R. Satchler, Angular Momentum (Clarendon Press, Oxford, 1975). 